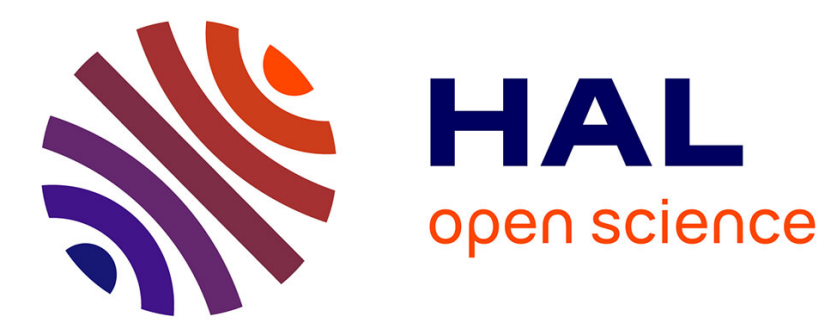

\title{
Faithful extensions on finite orders classes
}

\author{
Alain Guillet, Jimmy Leblet, Jean-Xavier Rampon
}

\section{To cite this version:}

Alain Guillet, Jimmy Leblet, Jean-Xavier Rampon. Faithful extensions on finite orders classes. The Australasian Journal of Combinatorics, 2017, 69 (1), pp.1-17. hal-01625566

\section{HAL Id: hal-01625566 https://hal.science/hal-01625566}

Submitted on 27 Oct 2017

HAL is a multi-disciplinary open access archive for the deposit and dissemination of scientific research documents, whether they are published or not. The documents may come from teaching and research institutions in France or abroad, or from public or private research centers.
L'archive ouverte pluridisciplinaire HAL, est destinée au dépôt et à la diffusion de documents scientifiques de niveau recherche, publiés ou non, émanant des établissements d'enseignement et de recherche français ou étrangers, des laboratoires publics ou privés. 


\title{
Faithful extension on finite order classes
}

\author{
Alain Guillet \\ SMCS, Université catholique Louvain \\ Voie du Roman Pays, 20 B-1348 Louvain-la-Neuve \\ Belgium \\ alain.guillet@uclouvain.be \\ JIMMY LEBLET \\ Centre de recherche Magellan \\ IAE Lyon, Université Jean Moulin Lyon 3 \\ 6 cours Albert Thomas, 69008 Lyon \\ France \\ jimmy.leblet@univ-lyon3.fr \\ JEAN-XAVIER RAMPON \\ FST de l'Université de Nantes \\ 2, rue de la Houssinière, BP 92208 44322 Nantes Cedex 3 \\ France \\ jean-xavier.rampon@univ-nantes.fr
}

\begin{abstract}
In the particular case of finite orders, we investigate the notion of faithful extension among relations introduced in 1971 by R. Fraïssé: an order $Q$ admits a faithful extension relative to an order $P$ if $P$ does not embed into $Q$ and there exists a strict extension of $Q$ into which $P$ still does not embed. For most of the known order classes, we prove that if $P$ and $Q$ belong to a class then $Q$ admits a faithful extension in this class. For the class of distributive lattices, we give an infinite family of orders $P$ and $Q$ such that $P$ does not embed into $Q$ and embeds in every strict extension of $Q$.
\end{abstract}

\section{Introduction}

We start by fixing some notation and definitions on orders. Notation and definitions which are specific to individual sections will be introduced as necessary. For further information, in particular for order classes that we mention but that we do not study, we refer to the books of Schröder [13] and of Trotter [14]. We use standard set 
theoretical notation. The cardinal of a set $A$ will be denoted $|A|$. Given any strictly positive integer $k$, the set $\{1, \ldots, k\}$ will be denoted by $[k]$.

A strict order, or simply an order in the sequel, $P$, is an ordered pair $\left(V(P),<_{P}\right)$, where its ground set $V(P)$ is a (finite) set and $<_{P}$ is an irreflexive, transitive (and thus antisymmetric) binary relation on $V(P)$. In the following, we assume that $x, y \in V(P)$ and $X \subseteq V(P)$. The notation $x \leq_{P} y$ signifies either $x<_{P} y$ or $x=y$, and the notation $x-<_{P} y$ signifies both $x<_{P} y$ and, for all $z \in V(P)$, if $x \leq_{P} z<_{P} y$ then $x=z$. In the latter case, $x$ is called a lower cover of $y$, and $y$ is called an upper cover of $x$.

When $x$ and $y$ are distinct, they are comparable (respectively, incomparable), denoted by $x \sim_{P} y$ (respectively, $x \|_{P} y$ ), if either $x<_{P} y$ or $y<_{P} x$ (respectively, neither $x<_{P} y$ nor $\left.y<_{P} x\right)$. The element $x$ is maximal (respectively, minimal) if for every $z \in V(P)$ we have that $x \leq_{P} z$ (respectively, $z \leq_{P} x$ ) implies $x=z$. The set of all maximal (respectively, minimal) elements of $\mathrm{P}$ is denoted by $\operatorname{Max}(P)$ (respectively, $\operatorname{Min}(P)$ ). The element $x$ is isolated if it is both a maximal and a minimal element. The set $X$ is a chain (respectively, an antichain) of $P$ when every two distinct elements of $X$ are comparable (respectively, incomparable). The rank of $x$ in $P$ denoted by $\operatorname{rank}_{P}(x)$ is the maximal cardinality minus one of a chain of $P$ having $x$ for greatest element. For example, $\operatorname{rank}_{P}(x)=0$ if and only if $x$ is a minimal element of $P$. For every natural number $k$, the set of all the elements of $P$ with rank $k$ is denoted by $\mathcal{R}_{P}(k)$, that is $\mathcal{R}_{P}(k)=\left\{x \in V(P): \operatorname{rank}_{P}(x)=k\right\}$. The height of $P$, denoted by $h(P)$, is the maximal cardinality minus one of a chain of $P$. For each element $X$ of $2^{V(P)}$ (that is, $X$ is a subset of $V(P)$ ) we associate the suborder $\left(X,(X \times X) \cap<_{P}\right)$ of $P$ induced by $X$ and denoted by $P[X]$. When $X=V(P)-\{x\}$ we will rather use the notation $P-x$.

Let us consider two orders $P$ and $Q$. An isomorphism from $P$ onto $Q$ is a bijective mapping $f: V(P) \longrightarrow V(Q)$ such that for all $x, y \in V(P), x<_{P} y$ if and only if $f(x)<_{Q} f(y)$. The order $P$ embeds into the order $Q$ if there exists a suborder of $Q$ isomorphic to $P$. The order $Q$ is an extension of $P$ whenever $P=Q[V(P)]$ (that implies $V(P) \subseteq V(Q))$. Consequently if $Q$ is an extension of $P$ then $P$ embeds into $Q$. An extension $Q$ of an order $P$ is strict if $|V(Q)|>|V(P)|$. If moreover, $|V(Q)|-|V(P)|=1, Q$ is then said to be a sharp extension of $P$.

The notion of faithful extension was first introduced for arbitrary relations in 1971 by Fraïssé [4]. A detailed study of this notion for relations and for bivalent tables is provided in the book of Fraïssé [5] (Chapter 8). For example, if $R$ is a finite structure, then the collection $\Omega_{\neg R}$ of finite structure $S$ such that $R$ does not embed into $S$ is an infinite age in the terminology of Fraïssé. Notice that such inextensibility properties were already used in 1948 by Fraïssé [3] for classifying relations and in 1968 by Jullien [11] in his theorem on words on finite alphabets. In 1993, Fraïssé and Hazim-Sharif [6] were interested in faithful extensions of finite orders: they said that an order $Q$ admits a faithful extension relative to an order $P$ when $(i) P$ does not embed into $Q$, and $(i i)$ there exists $Q^{+}$a sharp extension of $Q$ such that $P$ does not embed into $Q^{+}$. 
The problem of determining whether any pair of orders admit faithful extensions is quite obvious. Indeed, as indicated in [4], it is sufficient to notice that: if an order $P$ does not embed into an order $Q$, then for any $x \notin V(Q)$ either $P$ does not embed into $\left(V(Q) \cup\{x\},<_{Q}\right)$ or $P$ does not embed into $\left(V(Q) \cup\{x\},<_{Q} \cup\{(x, y): y \in V(Q)\}\right)$. Consequently, the notion of faithful extension becomes interesting only when it is restricted to subclasses of finite orders. Under these considerations, Fraïssé and Hazim-Sharif [6], following an idea of Bonnet, investigated some classes of fixed height orders. For that study, they introduced the notions of $h$-inextensibility and of h-extensibility:

- An order $P$ is h-inextensible for an order $Q$ if and only if $P$ and $Q$ have both height $h, P$ does not embed into $Q$, and $P$ embeds into every sharp extension of $Q$ of height $h$.

- An order $P$ is $h$-extensible if and only if for every order $Q$ of height $h$ so that $P$ does not embed into, there exists $Q^{+}$a sharp extension of $Q$ of height $h$ so that $P$ does not embed into $Q^{+}$.

If these three notions, faithful extension, $h$-inextensibility and $h$-extensibility, can be directly extended to any class of orders closed by suborders, in order to take into account any class of orders, we rather propose the following generalizations:

Definition 1.1 An order $Q$ admits a faithful extension relative to an order $P$ when (i) $P$ does not embed into $Q$ and (ii) there exists $Q^{\prime}$ a strict extension of $Q$ such that $P$ does not embed into $Q^{\prime}$.

Definition 1.2 An order $P$ is $\mathcal{C}$-inextensible whenever (i) $P$ belongs to $\mathcal{C}$ and (ii) there exists an order $Q$ in $\mathcal{C}$ into which $P$ does not embed, and such that $P$ embeds into every strict extension of $Q$ belonging to $\mathcal{C}$.

Definition 1.3 An order $P$ is $\mathcal{C}$-extensible whenever (i) $P$ belongs to $\mathcal{C}$ and (ii) every order $Q$ in $\mathcal{C}$, into which $P$ does not embed, admits a strict extension, belonging to $\mathcal{C}$, into which $P$ does not embed. Moreover, if every $P$ in $\mathcal{C}$ is $\mathcal{C}$-extensible then $\mathcal{C}$ is said to be extensible.

Let $\mathcal{H}_{k}$ be the height $k$ orders class, that is, the class of orders whose maximal cardinality chains contain $k+1$ elements. Let $\mathcal{I} s o l(P)$ be the set of isolated elements of an order $P$.

An element $x$ of an order $P$ is max dominating (respectively, min dominating) if it is strictly greater (respectively, strictly smaller) in $P$ than every element in $V(P)$ being not maximal (respectively, not minimal), equivalently $P-\left\{y \in V(P): y<_{P} x\right\}$ (respectively, $P-\left\{y \in V(P): x<_{P} y\right\}$ ) is an antichain. The results obtained by Fraïssé and Hazim-Sharif [6] can be expressed as: there exists $\mathcal{H}_{1}$-extensible orders, there exists $\mathcal{H}_{1}$-inextensible orders, and for $k \geq 2$, any order $P$ such that either $\mathcal{I}$ sol $(P)$ is empty or such that $P[V(P)-\mathcal{I}$ sol $(P)]$ does not admit a max dominating element, or such that $P[V(P)-\mathcal{I} s o l(P)]$ does not admit a min dominating element, is $\mathcal{H}_{k}$-extensible. 
In this paper we study the notion of faithful extension on various classes of orders. We present a result of inextensibility for the class of distributive lattices, and, we show that most of the known classes are extensible. In Section 2, we recall some known or easy facts about embeddings and about orders extensions. In Section 3, we show, for several of orders, that only two types of extensions are sufficient to ensure that they are extensible. Among them we have interval orders, k-dimensional orders, semi-orders, weak-orders, connected orders, lattices, and in-tree orders. In Section 4, we show that the class of distributive lattices admits an infinite family of inextensible orders. In Section 5, by using a variable number of extensions we prove that the class of disconnected orders, the class of width $k$ orders, and the class of indecomposable orders on at least 6 elements are all extensible. In Section 6, we conclude by partial results on the class $\mathcal{H}_{k}$ of height $k$ orders. Particularly, we show that an order $P$ is $\mathcal{H}_{k}$-extensible whenever there exists $i \in\{0, \ldots, k-1\}$ such that $\operatorname{Max}(P) \cap \mathcal{R}_{P}(i)=\emptyset$.

We finish this section by fixing some other notation and definitions that we use throughout the following. Given an order $P$, let $x, y \in V(P)$ and let $X \subseteq V(P)$. The predecessors set of $x$ is the set $D_{P}(x)=\left\{y \in V(P): y<_{P} x\right\}$ and the set of immediate predecessors of $x$ is the set $\left.D_{P}^{i m}(x)=\left\{y \in V(P): y<_{P} x\right)\right\}$. The same holds for the successors sets with $U_{P}(x)$ and $U_{P}^{i m}(x)$. The dual order of $P$ is the order $P^{d}$ where $V\left(P^{d}\right)=V(P)$ and $<_{P d}=\left\{(x, y) \in V(P) \times V(P):(y, x) \in<_{P}\right\}$. Given a family $\left(P_{i}\right)_{i \in I}$ of pairwise disjoint orders, that is, for $i, j \in I$ we have $V\left(P_{i}\right) \cap V\left(P_{j}\right)=\emptyset$ for all $i \neq j$, the parallel composition of the $P_{i}^{\prime}$ 's is the order $P=\bigoplus_{i \in I} P_{i}$ where $V(P)=\bigcup_{i \in I} V\left(P_{i}\right)$ and $<_{P}=\bigcup_{i \in I}<_{P_{i}}$. When $|I|=2$, with for example $I=[2]$, we rather use $P_{1} \oplus P_{2}$. Given $P_{1}$ and $P_{2}$, two disjoint orders, the left series composition of $P_{2}$ by $P_{1}$ or, equivalently, the right series composition of $P_{1}$ by $P_{2}$, is the order $P=P_{1} \otimes P_{2}$ where $V(P)=V\left(P_{1}\right) \cup V\left(P_{2}\right)$ and $<_{P}=<_{P_{1}} \cup<_{P_{2}} \cup\left(V\left(P_{1}\right) \times V\left(P_{2}\right)\right)$. When $P_{1}=(\{x\}, \emptyset)$ (respectively, $P_{2}=(\{y\}, \emptyset)$ ), we rather use $x \otimes P_{2}$ (respectively, $\left.P_{1} \otimes y\right)$. Given a mapping $\psi$ from a set $A$ into a set $B$, to simplify notation, we also use $\psi$ to denote the corresponding mapping from the subsets of $A$ into the subsets of $B$. That is, for $X \in 2^{A}$ and for $Y \in 2^{B}$, we have $\psi(X)=\{\psi(x): x \in X\}$ and $\psi^{-1}(Y)=\bigcup_{y \in Y} \psi^{-1}(y)$, where $\psi^{-1}(y)=\{x \in X: \psi(x)=y\}$. Also, when $\psi$ is injective (one-to-one), for every $b \in B$, whenever $\psi^{-1}(b)$ exists, we use indifferently $\psi^{-1}(b)$ either for the singleton or for the element itself.

\section{Preliminaries}

\subsection{Embedding}

We recall some known facts about embeddings and we provide some proofs for the reader's convenience.

Fact 2.1 Let $P$ and $Q$ be two orders such that $P$ embeds into $Q$ by $\psi$. Then for every $x \in V(Q)$ in the range of $\psi$, we have firstly that $P\left[D_{P}\left(\psi^{-1}(x)\right)\right]$ embeds into $Q\left[D_{Q}(x)\right]$ by $\psi$ restricted to $D_{P}\left(\psi^{-1}(x)\right)$ and secondly that $D_{P}\left(\psi^{-1}(x)\right)=\psi^{-1}\left(D_{Q}(x)\right)$. The same holds for the successors sets. 
From this fact the next property follows immediately:

Property 2.1 Let $P$ and $Q$ be two orders such that $P$ embeds into $Q$ by $\psi$. Let $x, y \in V(Q)$ in the range of $\psi$; then we have:

(i) $x<_{Q} y$ implies that $\psi^{-1}(x)<_{P} \psi^{-1}(y)$.

(ii) $\left|\psi^{-1}\left(D_{Q}^{i m}(x)\right)\right|=\left|D_{Q}^{i m}(x)\right|$ implies that $D_{P}^{i m}\left(\psi^{-1}(x)\right)=\left\{\psi^{-1}(z): z \in D_{Q}^{i m}(x)\right\}$, and $\left|\psi^{-1}\left(U_{Q}^{i m}(x)\right)\right|=\left|U_{Q}^{i m}(x)\right|$ implies that $U_{P}^{i m}\left(\psi^{-1}(x)\right)=\left\{\psi^{-1}(z): z \in U_{Q}^{i m}(x)\right\}$.

(iii) $x \in \operatorname{Max}(Q)$ (respectively, $x \in \operatorname{Min}(Q)$ ) implies that $\psi^{-1}(x) \in \operatorname{Max}(P)$ (respectively, $\left.\psi^{-1}(x) \in \operatorname{Min}(P)\right)$.

(iv) if $x$ is the least (respectively, greatest) element of $Q$, then $\psi^{-1}(x)$ is the least (respectively, greatest) element of $P$.

Proof. Assertion (i): firstly, notice that for any order $H$ we have that $a<_{H} b$ if and only if both $a<_{H} b$ and $U_{H}(a) \cap D_{H}(b)=\emptyset$. Secondly, recall that for any mapping $\varphi$ from $U$ to $V$ and for any subsets $A, B$ of $V$, we have that $\varphi^{-1}(A) \cap \varphi^{-1}(B)=$ $\varphi^{-1}(A \cap B)$. Then this case immediately follows from Fact 2.1.

Assertion (ii): Since $\left|\psi^{-1}\left(D_{Q}^{i m}(x)\right)\right|=\left|D_{Q}^{i m}(x)\right|$ we have that $D_{Q}^{i m}(x) \subseteq \psi(V(P))$. Consequently, by Assertion (i), it follows that $\left\{\psi^{-1}(z): z \in D_{Q}^{i m}(x)\right\} \subseteq D_{P}^{i m}\left(\psi^{-1}(x)\right)$. Assume that there exists $t \in D_{P}^{i m}\left(\psi^{-1}(x)\right) \backslash\left\{\psi^{-1}(z): z \in D_{Q}^{i m}(x)\right\}$. Then we have $\psi(t)<_{Q} \psi\left(\psi^{-1}(x)\right)=x$, and thus there exists $\alpha \in V(Q)$ such that $\psi(t)<_{Q} \alpha-<_{Q} x$. Consequently, we obtain that $t=\psi^{-1}(\psi(t))<_{P} \psi^{-1}(\alpha)<_{P} \psi^{-1}(x)$ : a contradiction. The dual case follows the same lines.

Assertion (iii): since for any $x \in \operatorname{Max}(Q)$ (respectively, $x \in \operatorname{Min}(Q))$ holds $U_{Q}(x)=\emptyset$ (respectively, $D_{Q}(x)=\emptyset$ ) then the result directly follows from Fact 2.1.

Assertion (iv): for every $y \in V(Q)-\{x\}$, we have that $x<_{Q} y$ (respectively, $y<_{Q} x$ ). For every $z \in V(P)-\left\{\psi^{-1}(x)\right\}$, we have that $\psi(z) \in V(Q)-\{x\}$. Consequently, by Fact 2.1, holds $\psi^{-1}(x) \in D_{P}(z)$ (respectively, $\psi^{-1}(x) \in U_{P}(z)$ ).

\subsection{Sharp extension}

Most of the time, sharp extensions are sufficient to establish or to confirm the existence of faithful extensions. In this section, we detail two of them which are particularly useful.

Let $Q$ and $P$ be two orders such that $V(P) \cap V(Q)=\{y\}$. We define the order $Q_{y}^{P}$ by (i) $V\left(Q_{y}^{P}\right)=V(Q) \cup V(P)$, and (ii) for all $a \neq b \in V\left(Q_{y}^{P}\right)$ we have $a<_{Q_{y}^{P}} b$ whenever either $\left(\{a, b\} \subseteq V(P)\right.$ and $\left.a<_{P} b\right)$ or $\left(\{a, b\} \subseteq V(Q)\right.$ and $\left.a<_{Q} b\right)$ or $\left(a \in V(P), b \in(V(Q)-V(P))\right.$ and $\left.y<_{Q} b\right)$ or $(b \in V(P), a \in(V(Q)-V(P))$ and $\left.a<_{Q} y\right)$. This order is said to be obtained by substitution of $y$ by $P$ in $Q$.

Fact 2.2 Let $P$ and $Q$ be two orders such that $V(P) \cap V(Q)=\{y\}$. Then $Q_{y}^{P}$ is a sharp extension of $Q$ whenever $|V(P)|=2$. Moreover, with $V(P)=\{y, x\}$ we have that $Q_{y}^{P}-x=Q$ and that $Q_{y}^{P}-y$ is isomorphic to $Q$. 

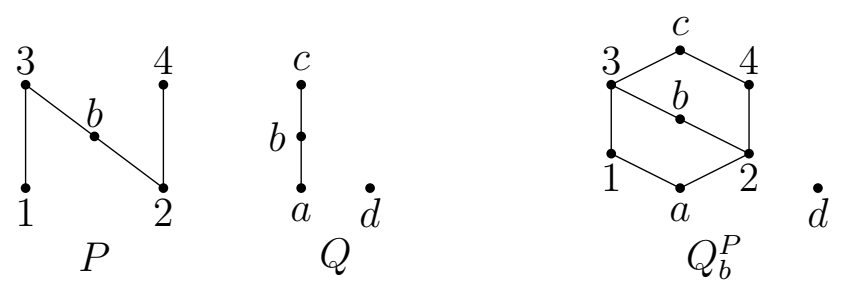

Figure 1: The substitution of $b$ by $P$ in $Q$.

Lemma 2.1 Let $P$ and $Q$ be two orders such that $P$ does not embed into $Q$. Let $x \notin V(Q)$, let $y \in V(Q)$ and let $Z$ be any order such that $V(Z)=\{x, y\}$. If $P$ embeds by $\psi$ into $Q_{y}^{Z}$ then both $\psi^{-1}(x)$ and $\psi^{-1}(y)$ exist.

Proof. Since $P$ does not embed into $Q$ then $\psi^{-1}(x)$ exists. Now, assume that $\psi^{-1}(y)$ does not exist. Thus, $P$ embeds into $Q_{y}^{Z}-y$, which is in contradiction with the fact that $Q_{y}^{Z}-y$ is isomorphic to $Q$.

Property 2.2 Let $P$ and $Q$ be two orders such that $Q$ has a greatest element and $P$ does not embed into $Q$. If $P$ embeds into $Q \otimes x$, for $x \notin V(Q)$, then $P$ has a greatest element with a unique immediate predecessor.

Proof. Let $\top_{Q}$ be the greatest element of $Q$, and assume that $P$ embeds into $Q \otimes x$ by $\psi$. First of all, notice that $Q \otimes x=Q_{\top_{Q}}^{Z}$ where $Z=\left(\left\{\top_{Q}, x\right\},\left\{\left(\top_{Q}, x\right)\right\}\right)$. Now, by Lemma 2.1, we have that both $\psi^{-1}(x)$ and $\psi^{-1}\left(T_{Q}\right)$ exist. From Property 2.1 (iv) it follows that $\psi^{-1}(x)$ is the greatest element of $P$. Now, since $D_{Q \otimes x}^{i m}(x)=\left\{\top_{Q}\right\}$, for all $p \in\left(V(P) \backslash\left\{\psi^{-1}(x)\right\}\right)$ we have that $\psi(p) \leq_{Q \otimes x} \top_{Q}$, and thus that $D_{P}^{i m}\left(\left(\psi^{-1}(x)\right)\right)=$ $\left\{\psi^{-1}\left(\top_{Q}\right)\right\}$.

\section{Some easy extensibility results}

In this section, in order to show that the classes that we investigate are extensible, we only have to exhibit two types of sharp extension per class.

\subsection{Parallels and series extensions}

The underlying idea of the argumentation developed to establish that any order is $\mathcal{O}$-extensible - with $\mathcal{O}$ the class of all orders- deserves to be expressed in a proposition that we attribute to Fraïssé:

Proposition 3.1 [Fraïssé] Let $\mathcal{C}$ be an order class stable by the parallel composition with a one element order and stable by the left or right series composition by a one element order. Then any order in $\mathcal{C}$, on at least two elements, is $\mathcal{C}$-extensible. 
Proof. Let $P, Q$ be two orders belonging to $\mathcal{C}$ with $|V(P)| \geq 2$ and such that $P$ does not embed into $Q$. Let $x \notin V(Q)$. First, assume that $P$ embeds into $Q \oplus x$ by $\psi$. Then, since $P$ does not embed into $Q$, we have that $\psi^{-1}(x)$ exists and thus, by Fact 2.1 , we have that $\psi^{-1}(x)$ is an isolated element in $P$. Second, assume, for example, that $P$ embeds into $Q \otimes x$ by $\phi$. Again, since $P$ does not embed into $Q$, we have that $\phi^{-1}(x)$ exists and thus, by Property 2.1 (iv), we obtain that $P$ has a greatest element. Thus, if we assume that $P$ embeds both into $Q \oplus x$ and into $Q \otimes x$ it follows that $P$ must have a unique element: a contradiction. The case $P$ embeds into $x \otimes Q$ follows exactly the same lines.

As an immediate consequence we obtain that the classes of $\mathrm{N}$-free orders (see Schröder [13] on page 50), series-parallel orders (see Schröder [13] on page 220), threshold orders, truncated lattices (see Schröder [13] on page 137), forest of intree orders (or CS-forest see Trotter [14] on page 116), $k$-dimensional orders with $k \geq 2$ (see Schröder [13] on page 169 or Trotter [14] on page 9), interval orders (see Schröder [13] on page 186 or Trotter [14] on page 86), cycle-free orders (see Trotter [14] on page 123), and decomposable orders (see Schröder [13] on page 203 or Trotter [14] on page 24) are extensible.

\subsection{Min-Max substitution and series extensions}

In the same vein as Fraïssé's proposition, we have:

Proposition 3.2 Let $\mathcal{C}$ be an order class stable by the right (respectively, the left) series composition by a one element order, and stable by the substitution of any of its maximal (respectively, minimal) elements by a two elements antichain. Then any order in $\mathcal{C}$ is $\mathcal{C}$-extensible.

Proof. Let $P, Q$ be two orders belonging to $\mathcal{C}$ such that $P$ does not embed into $Q$. Let $x \notin V(Q)$. First, assume that $P$ embeds into $Q \otimes x$ by $\phi$. Then, since $P$ does not embed into $Q$, we have that $\phi^{-1}(x)$ exists and thus, by Property 2.1 (iv), we obtain that $P$ has a greatest element. Second, let $y \in \operatorname{Max}(Q)$, let $Z=(\{y, x\}, \emptyset)$ and assume that $P$ embeds into $Q_{y}^{Z}$ by $\psi$. Then, by Lemma $2.1, \psi^{-1}(x)$ and $\psi^{-1}(y)$ exist and moreover, by Property 2.1 (iii), they belong to $\operatorname{Max}(P)$. Thus assuming that $P$ embeds both into $Q \otimes x$ and into $Q_{y}^{Z}$ leads to a contradiction.

The case $P$ embeds both into $x \otimes Q$ and into $Q_{y}^{Z}$, for $y \in \operatorname{Min}(Q)$, follows exactly the same lines.

First, notice that, if $\mathcal{C}$ is a class of orders which satisfies the conditions of Proposition 3.2, then its restriction, on connected orders, also satisfies these conditions. Now, as an immediate consequence of Proposition 3.2, we obtain that the classes of weak orders (or weakly ordered set see Schröder [13] on page 181), semi-orders (see Schröder [13] on page 197 or Trotter [14] on page 192), and connected orders on at least two elements (see Schröder [13] on page 44 or Trotter [14] on page 4) are extensible. Also, the class of chain dominated orders, introduced by Guenver et al. [8], that is the class of orders which have a chain such that every element of the order either belongs to this chain or has all its covers in this chain, is then clearly extensible. 


\subsection{Minimal pendant and series extensions}

Recall that an order $P$ is an in-tree order if it has a greatest element and for every $x \in V(P)$ the set $U_{P}(x)$ is a chain. That is, an in-tree order is any order whose transitive reduction is an anti-arborescence.

For an order $P$, having $\top_{P}$ has greatest element, and for an element $x \notin V(P)$, we denote its minimal pendant extension by the order $P \otimes x=\left(V(P) \cup\{x\},<_{P}\right.$ $\left.\cup\left\{\left(x, \top_{P}\right)\right\}\right)$. Obviously, if $P$ is an in-tree order then $P \otimes x$ still belongs to this class.
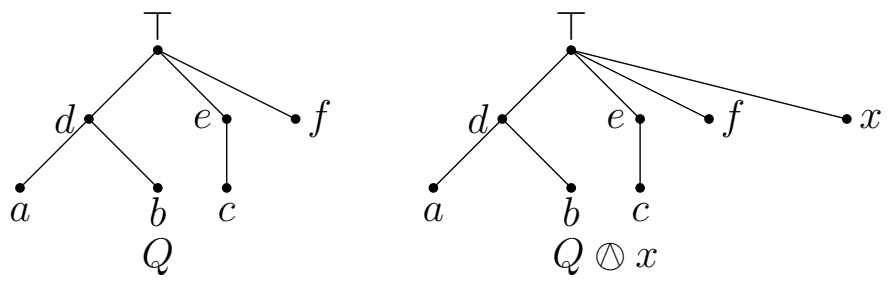

Figure 2: The minimal pendant extension.

Proposition 3.3 Let $\mathcal{C}$ be the class of in-tree orders. Then any order $P$ in $\mathcal{C}$, having at least three elements, is $\mathcal{C}$-extensible.

Proof. Let $P$ be an in-tree order on at least 3 elements, let $Q$ be an in-tree order into which $P$ does not embed and let $x \notin V(Q)$. First, assume that $P$ embeds into $Q \otimes x$. Then, by Property 2.2 , we obtain that $\left|D_{P}^{i m}\left(\top_{P}\right)\right|=1$. Second, assume that $P$ embeds into $Q \otimes x$ by $\psi$. Then, since $P$ does not embed into $Q$, we have that $\psi^{-1}(x)$ exists. But, since $U_{Q \otimes x}(x)=\left\{\top_{Q \otimes x}\right\}$ and since $P$ has at least 2 elements, the fact that $P$ has a greatest element together with Fact 2.1 imply that $\psi^{-1}\left(\top_{Q \otimes x}\right)$ exists. Moreover, Property 2.1 (iv) insures that $\psi^{-1}\left(\top_{Q \otimes x}\right)=\top_{P}$. Finally, since $x<_{Q \otimes x} \top_{Q \otimes x}$, Property 2.1 (i) implies that $\psi^{-1}(x)-<_{P} \top_{P}$. Consequently, since $\top_{P}$ has a unique immediate predecessor, we obtain that $P$ is isomorphic to the 2 elements chain: this contradicts that $|V(P)| \geq 3$.

\subsection{Universal complement and series extensions}

Following Birkhoff [1] (on page 6), a lattice is an order $L$ any two of whose elements have a greatest lower bound or "meet" denoted by $x \wedge_{L} y$, and a least upper bound or "join" denoted by $x \vee_{L} y$.

For any order $P$, we denote $\top_{P}$, respectively, $\perp_{P}$, the greatest, respectively, the least, element of $P$ when it exists. For any order $P$, on at least two elements, having both a greatest and a least element, and for any $x \notin V(P)$, we denote by $P \diamond x$ the order $\left(V(P) \cup\{x\},<_{P} \cup\left\{\left(x, \top_{P}\right),\left(\perp_{P}, x\right)\right\}\right)$. Obviously, for any lattice $Q$, on at least two elements, and for any element $x \notin V(Q)$, the order $Q \diamond x$ is still a lattice.

Proposition 3.4 Let $\mathcal{C}$ be the class of lattices. Then any order $P$ in $\mathcal{C}$, having at least four elements, is $\mathcal{C}$-extensible. 


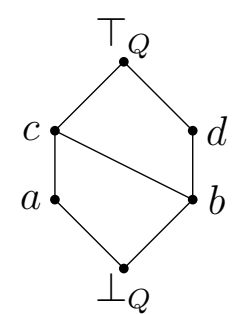

$Q$

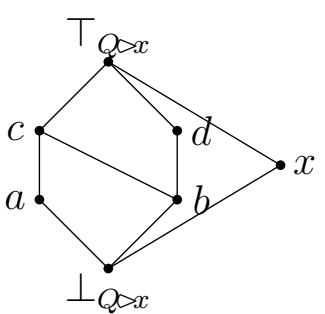

$Q \triangleright x$

Figure 3: The universal complement extension.

Proof. Let $P$ and $Q$ be two lattices such that $P$ does not embed into $Q,|V(P)| \geq 4$ and $x \notin V(Q)$. First, assume that $P$ embeds into $Q \otimes x$ then, by Property 2.2, we obtain that $\left|D_{P}^{i m}\left(\top_{P}\right)\right|=1$. Second, assume that $P$ embeds into $Q \diamond x$ by $\psi$. Then, since $P$ does not embed into $Q$, we have that $\psi^{-1}(x)$ exists. Since $D_{Q \triangleright x}(x)=\left\{\perp_{Q \triangleright x}\right\}$ and $U_{Q \triangleright x}(x)=\left\{\top_{Q \triangleright x}\right\}$, then, since $P$ is a lattice on at least three elements, we have by Fact 2.1 that both $\psi^{-1}\left(\top_{Q \triangleright x}\right)$ and $\psi^{-1}\left(\perp_{Q \triangleright x}\right)$ exist. By Property 2.1 (iv) we have that $\psi^{-1}\left(\top_{Q \triangleright x}\right)=\top_{P}$ and $\psi^{-1}\left(\perp_{Q \triangleright x}\right)=\perp_{P}$. Now, since $\perp_{Q \triangleright x}<_{Q \triangleright x} x<_{Q \triangleright x} \top_{Q \triangleright x}$, Property 2.1 (i) implies that $\perp_{P}<_{P} \psi^{-1}(x)<_{P} \top_{P}$. Finally the fact that $\top_{P}$ has a unique immediate predecessor implies that $P$ is isomorphic to the 3 elements chain: this contradicts that $|V(P)| \geq 4$.

Remark 3.1 Given a planar lattice $L$, since for $x \notin V(L)$ we have that both $L \otimes x$ and $L \diamond x$ are obviously planar lattices; this proposition remains true when $\mathcal{C}$ is the class of planar lattices.

\section{An inextensibility result}

In Section 3.4 we show that lattices are extensible. However, this extensibility result is no longer possible for distributive lattices. We even prove that the simplest family of lattices provides an infinite family of inextensible distributive lattices. To that purpose, we first recall G. Birkhoff's characterization of distributive lattices by forbidden sublattices (see for example Davey and Priestley [2] Theorem 6.10 for a proof).

Theorem 4.1 A lattice is distributive if and only if none of its sublattices is isomorphic to $M_{3}$ or $N_{5}$.

Since $\mathcal{B}^{k}$, the boolean lattice with $k$ atoms, is for, $k \in \mathbb{N}^{*}$, isomorphic to $\left(2^{[k]}, \subseteq\right)$ (see for example Davey and Priestley [2] Theorem 8.3 for a proof), by aim of simplicity, in the sequel we consider that $\mathcal{B}^{k}=\left(2^{[k]}, \subseteq\right)$.

Concerning the extensions of boolean lattices, first we notice that any strict extension of $\mathcal{B}^{1}$, being a lattice, contains the 3 -elements chain as suborder. For the other boolean lattices, if the number of strict extensions, being a lattice, increases with the number of atoms, however, none of them are distributive when we keep the height constant: 


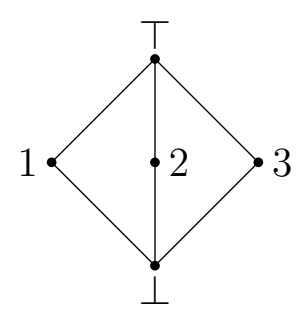

$M_{3}$

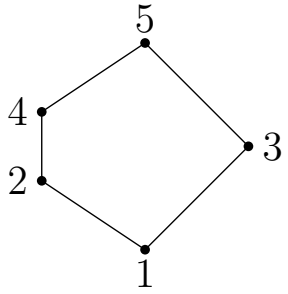

$N_{5}$

Figure 4: The forbidden sublattices in distributive lattice.

Theorem 4.2 A finite boolean lattice has no strict extension, of the same height, which is a distributive lattice.

Proof. The case $\mathcal{B}^{1}$ being already discussed, we consider $\mathcal{B}^{k}$ for $k \geq 2$. Now, let $L$ be any strict extension, of $\mathcal{B}^{k}$, having the same height and being a lattice. First, since $\mathcal{B}^{k}$ embeds in $L$ and since $h(L)=h\left(\mathcal{B}^{k}\right)$ it follows that $\top_{L}=\top_{\mathcal{B}^{k}}$ and that $\perp_{L}=\perp_{\mathcal{B}^{k}}$. Consequently, for any $x \in\left(V(L) \backslash 2^{[k]}\right)$, there exists $A, B \in 2^{[k]}$, such that $A<_{L} x<_{L} B$. Among all such $(A, x, B) 3$-uplets, takes one of them such that $h\left(\mathcal{B}^{k}\left[[A, B]_{\mathcal{B}^{k}}\right]\right)$ is minimal, when as usually, $[A, B]_{\mathcal{B}^{k}}=\left\{Z \in 2^{[k]}: A \subseteq Z \subseteq B\right\}$. Notice that $h(L)=h\left(\mathcal{B}^{k}\right)$ implies that $h\left(\mathcal{B}^{k}\left[[A, B]_{\mathcal{B}^{k}}\right]\right) \geq 2$.

- Assume that $h\left(\mathcal{B}^{k}\left[[A, B]_{\mathcal{B}^{k}}\right]\right)=2$, then $B=A \cup\{\alpha, \beta\}$ with $\alpha, \beta \in[k]$. Consequently, since $h(L)=h\left(\mathcal{B}^{k}\right)$, the set $\{A, A \cup\{\alpha\}, A \cup\{\beta\}, x, B\}$ induces a sublattice of $L$ isomorphic to $M_{3}$. Firstly, the set $\{A, A \cup\{\alpha\}, A \cup\{\beta\}, x, B\}$ induces a suborder of $L$ isomorphic to $M_{3}$. On the one hand, $\mathcal{B}^{k}$ is a suborder of $L$. On the other hand, if, for example, $x<_{L} A \cup\{\alpha\}$ then we have that $A<_{L} x<_{L}(A \cup\{\alpha\})$, and thus $h\left(\mathcal{B}^{k}\right)<h(L)$ : the remaining cases are proved under the same lines. Secondly, the set $\{A, A \cup\{\alpha\}, A \cup\{\beta\}, x, B\}$ actually induces a sublattice of $L$. If, for example, $(A \cup\{\alpha\}) \vee_{L} z=y$ with $z \in\{A \cup\{\beta\}, x\}$ and $y \neq B$ then we have $y<_{L} B$. Now, since $\mathcal{B}^{k}$ is a suborder of $L$, then $y \in\left(V(L) \backslash 2^{[k]}\right)$ and thus $(A \cup\{\alpha\})<_{L} y<_{L} B=(A \cup\{\alpha, \beta\})$. The remaining cases are proved under the same lines.

- Assume that $h\left(\mathcal{B}^{k}\left[[A, B]_{\mathcal{B}^{k}}\right]\right) \geq 3$, then there exists $C, D \in 2^{[k]}$, such that $A \subsetneq C \subsetneq D \subsetneq B$. Consequently, due to the minimality of $h\left(\mathcal{B}^{k}\left[[A, B]_{\mathcal{B}^{k}}\right]\right)$, the set $\{A, C, D, x, B\}$ induces a sublattice of $L$ isomorphic to $N_{5}$. Firstly, the set $\{A, C, D, x, B\}$ induces a suborder of $L$ isomorphic to $N_{5}$ : on the one hand $\mathcal{B}^{k}$ is a suborder of $L$ and, on the other hand, if, for example, $x<_{L} D$ then the 3 -uplet $(A, x, D)$ contradicts the choice of $(A, x, B)$, since $h\left(\mathcal{B}^{k}\left[[A, D]_{\mathcal{B}^{k}}\right]\right)<$ $h\left(\mathcal{B}^{k}\left[[A, B]_{\mathcal{B}^{k}}\right]\right)$. Secondly, the set $\{A, C, D, x, B\}$ actually induces a sublattice of $L$ : if, for example, $x \vee_{L} z=y$ with $z \in\{C, D\}$ and $y \neq B$ then we have $y<_{L} B$. Now, either $y \in 2^{[k]}$ and then the 3 -uplet $(A, x, y)$ contradicts the choice of $(A, x, B)$, or $y \in\left(V(L) \backslash 2^{[k]}\right)$ and then the 3-uplet $(z, y, B)$ contradicts the choice of $(A, x, B)$. The remaining cases are proved under the same lines. 
Let $\mathcal{D} \mathcal{L}$ be the class of distributive lattices, and let $C_{k}$ be the chain order on $k$ elements. Since the height of the boolean lattice $\mathcal{B}^{k}$ is $k$, as an immediate consequence of Theorem 4.2, we obtain:

Corollary 4.1 for all $k \in \mathbb{N}^{*}$, the chain $C_{k+2}$ is $\mathcal{D} \mathcal{L}$-inextensible.

Notice that this approach is no more possible for the class of modular lattices. Even though, for every $k \geq 2$ there exists a strict extension of $\mathcal{B}^{k}$, with $2^{k-1}$ more elements, being a modular lattice and in which $C_{k+2}$ still does not embed. We do believe that faithful extensions, for the class of modular lattices, are worth studying. Moreover, we think that this study should be inclined towards an extensibility result.

\section{Some more extensibility results}

In this section, the number of sharp extensions, used to show that a class is extensible, is no more the same for all the elements of the class, but depends on structural parameters of the order to be extended.

\section{$5.1 \quad$ Disconnected orders}

Recall that a disconnected order is any order whose comparability graph is disconnected. Using one more sharp extension as the number of connected components of the order to be extended, we show that the class of disconnected orders is extensible.
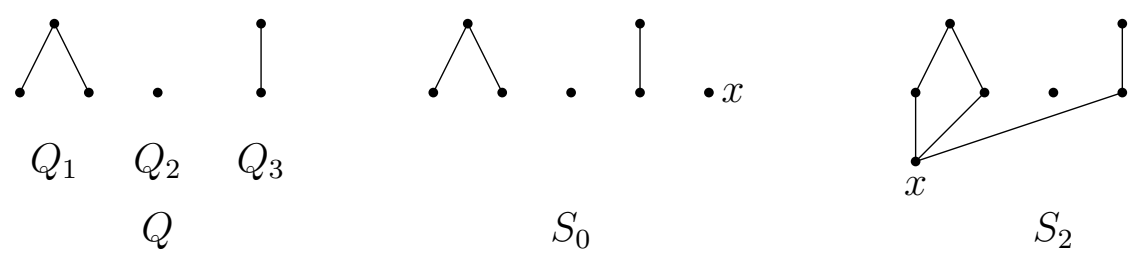

Figure 5: Some extensions used in the proof of Theorem 5.1.

Theorem 5.1 The class of disconnected orders is extensible.

Proof. Let $P$ and $Q$ be two disconnected orders. Then we have that $P=\bigoplus_{i \in[n]} P_{i}$ with $2 \leq n, Q=\bigoplus_{j \in[m]} Q_{j}$ with $2 \leq m$, and that all the $P_{i}$ 's and all the $Q_{j}$ 's are connected orders on at least one element. Assume that $P$ does not embed into $Q$ and let $x \notin V(Q)$. Let $S_{0}=\left(V(Q) \cup\{x\},<_{Q}\right)$, and for $j \in[m]$ let $S_{j}=\left(V(Q) \cup\{x\},<_{Q}\right.$ $\left.\cup\left\{(x, y): y \in V(Q)-V\left(Q_{j}\right)\right\}\right)$. Notice that all the $S_{j}$ 's are obviously sharp extensions of $Q$, and that, moreover, for every $j \in[m]$ the order $S_{j}\left[V(Q)-V\left(Q_{j}\right)\right]$ has $x$ for least element.

We claim that there exists $j \in\{0\} \cup[m]$ such that $P$ still does not embed into $S_{j}$. On the contrary, assume that, for every $j \in\{0\} \cup[m], P$ embeds into $S_{j}$ by $\phi_{j}$. Notice that since $P$ does not embed into $Q$ then, for every $j \in\{0\} \cup[m]$, we have that $\phi_{j}^{-1}(x) \in V(P)$. Consequently, for every $j \in[m]$, there exists a unique $i_{j} \in[n]$ 
such that $\phi_{j}\left(V\left(P_{i_{j}}\right)\right) \cap\left(V(Q)-V\left(Q_{j}\right)\right) \neq \emptyset$. Let us consider $S_{m}$, we can assume -up to a permutation- that $i_{m}=n$.

First, assume that there exists $j \in[m-1]$ such that $i_{j} \in[n-1]$. Thus, since $\bigoplus_{i \in[n-1]} P_{i}$ embeds into $Q_{m}$ by the appropriate restriction of $\phi_{m}$, and since $P_{n}$ embeds into $Q_{j}$ by the appropriate restriction of $\phi_{j}$, we immediately obtain an embedding of $P$ into $Q_{m} \oplus Q_{j}$ and thus into $Q$ which is a contradiction.

Consequently, we have that for every $j \in[m], i_{j}=n$ and thus, for every $j \in[m]$ we have that $\bigoplus_{i \in[n-1]} P_{i}$ embeds into $Q_{j}$ by the appropriate restriction of $\phi_{j}$. Now, consider $S_{0}$. Then either there exists $\alpha \in[m]$ such that $P_{n}$ embeds into $Q_{\alpha}$ with the appropriate restriction of $\phi_{0}$. Thus, for any $\beta \in[m]$ with $\beta \neq \alpha$, we immediately obtain an embedding of $P$ into $Q_{\alpha} \oplus Q_{\beta}$ : a contradiction. Or, $P_{n}$ embeds into $(\{x\}, \emptyset)$, by the appropriate restriction of $\phi_{0}$, and, thus, $P_{n}$ embeds into $Q_{i}$ for every $i \in[\mathrm{m}]$. Consequently, for every $\alpha, \beta \in[m]$ with $\beta \neq \alpha$, we obtain an embedding of $P$ into $Q_{\alpha} \oplus Q_{\beta}$ : a contradiction.

\subsection{Fixed width orders}

Recall that the width of an order $P$, denoted by $w(P)$, is the maximal cardinality of an antichain of $P$. Using at least as many sharp extensions as the height of the order to be extended, we show that the class of width $k$ orders is extensible for any $k \geq 2$.

Theorem 5.2 The class of width $k$ orders, with $2 \leq k$, is extensible.

Proof. Let $P$ and $Q$ be two orders such that $w(P)=w(Q)=k$ and $2 \leq k$. Assume that $P$ does not embed into $Q$, let $x \notin V(Q)$ and, for every $y \in V(Q)$, let $Z_{y}=(\{y, x\},\{(y, x)\})$. Notice that for every $y \in V(Q)$ we have that $w\left(Q_{y}^{Z_{y}}\right)=k$. To prove the theorem by contradiction we show that if $P$ embeds into every sharp extension of $Q$ then $w(P)=1$. For that purpose we show, by induction, that for every $0 \leq i \leq h(P),\left|\mathcal{R}_{P}(i)\right|=1$. The fact that $\left|\mathcal{R}_{P}(0)\right|=1$ is an immediate consequence of Property 2.1 (iv) when considering the sharp extension $x \otimes Q$. Consider $0 \leq n<h(P)$ and assume that for every $0 \leq j \leq n$ we have that $\mathcal{R}_{P}(j)=\left\{c_{j}\right\}$. Let $y \in \mathcal{R}_{Q}(n)$. Notice that $\mathcal{R}_{Q}(n) \neq \emptyset$ since $h(P)>n$ and $P$ embeds into every sharp extension of $Q$. Now, assume that $P$ embeds into $Q_{y}^{Z_{y}}$ by $\varphi$, and recall that, by Lemma 2.1, we have that both $\varphi^{-1}(y)$ and $\varphi^{-1}(x)$ exist. Now, since $y \in \mathcal{R}_{Q_{y}^{Z_{y}}}(n)$, it follows, by Fact 2.1 , that $\varphi^{-1}(y) \in\left\{c_{0}, \ldots, c_{n}\right\}$.

First, assume that $\varphi^{-1}(y)=c_{j}$ with $j<n$, then by Property 2.1 (i) it follows that $\varphi^{-1}(x)=c_{j+1}$. Consequently, using Fact 2.1, we obtain that $P\left[U_{P}\left[c_{j+1}\right]\right]$ embeds into $Q_{y}^{Z_{y}}\left[U_{Q_{y}^{Z_{y}}}[x]\right]$. Then, since $Q_{y}^{Z_{y}}\left[U_{Q_{y}^{Z_{y}}}[x]\right]$ is clearly isomorphic to $Q\left[U_{Q}[y]\right]$, it follows that $P\left[U_{P}\left[c_{j+1}\right]\right]$ embeds into $Q\left[U_{Q}[y]\right]$. Recall that $P=P\left[\left\{c_{0}, \ldots, c_{j}\right\}\right] \otimes P\left[U_{P}\left[c_{j+1}\right]\right]$ with $P\left[\left\{c_{0}, \ldots, c_{j}\right\}\right]$ being a chain. Then the fact that $y \in \mathcal{R}_{Q}(n)$ with $j<n$, immediately implies that $P$ embeds into $Q$ : a contradiction.

Consequently, we have that $\varphi^{-1}(y)=c_{n}$. Then, as an immediate consequence of Property 2.1 (i) and (ii), we obtain that $U_{P}^{i m}\left(c_{n}\right)=\left\{\varphi^{-1}(x)\right\}$, and, thus it immediately follows that $\mathcal{R}_{P}(n+1)=\left\{\varphi^{-1}(x)\right\}$. 


\subsection{Indecomposable orders}

Given an order $P$, a subset $X$ of $V(P)$ is an interval, or an autonomous set, of $P$ provided that for all $u, v \in X$ and $x \in V(P)-X,(u, x) \in<_{P}$ if and only if $(v, x) \in<_{P}$, and $(x, u) \in<_{P}$ if and only if $(x, v) \in<_{P}$. The trivial intervals of $P$ are : $\emptyset, V(P)$ and $\{x\}$ for every $x \in V(P)$. Then an order is indecomposable, or prime, if it has at least 3 elements and if all of its intervals are trivial: notice that this implies that it has in fact at least 4 elements. For further details about these notions, due to T. Gallai [7], we refer to Habib [9], Kelly [12] and to Ille [10].

Sharp extensions were obtained by mean of substitution. These substitutions consist of adding intervals, and thus do not preserve indecomposability. We give another process which preserves it: for an order $Q$, for $x \notin V(Q)$ and for any $m \in \operatorname{Max}(Q)$ (respectively, $m \in \operatorname{Min}(Q)$ ) we denote by $Q^{x, m}$ (respectively, $Q_{x, m}$ ) the order $\left(V(Q) \cup\{x\},<_{Q} \cup\{(y, x): y \in V(Q)-\{m\}\}\right)$ (respectively, $\left(V(Q) \cup\{x\},<_{Q}\right.$ $\cup\{(x, y): y \in V(Q)-\{m\}\}))$.
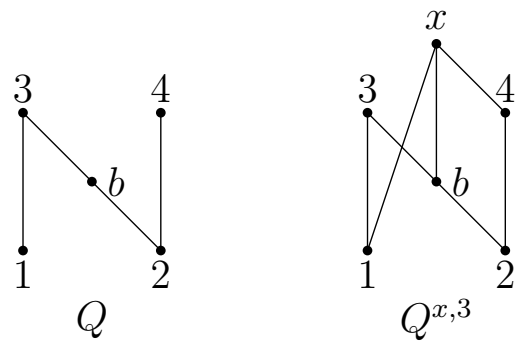

Figure 6: The $Q^{x, m}$ indecomposable sharp extension of Lemma 5.1.

Lemma 5.1 Let $Q$ be an indecomposable order and let $x \notin V(Q)$. Then:

(i) for every $m \in \operatorname{Max}(Q)$, having a predecessors set of maximal cardinality, the order $Q^{\prime}=Q^{x, m}$ is indecomposable,

(ii) for every $m \in \operatorname{Max}(Q)$, such that for every $t \in D_{Q}^{i m}(m) \cap \operatorname{Min}(Q)$ we have $U_{Q}(t) \neq\{m\}$, the order $Q^{\prime}=\left(V(Q) \cup\{x\},<_{Q} \cup\{(x, m)\}\right)$ is indecomposable,

(iii) for every $p \in \operatorname{Min}(Q)$, such that $\left|U_{Q}(p)\right|=1$, the order $Q^{\prime}=\left(V(Q) \cup\{x\},<_{Q}\right.$ $\cup\{(p, x)\})$ is indecomposable.

Proof. We proceed by contradiction. Let $X$ be a non-trivial interval of $Q^{\prime}$, that is $X \subsetneq V\left(Q^{\prime}\right)$ and $2 \leq|X|$. Since $Q=Q^{\prime}[V(Q)]$, and since $Q$ is indecomposable, it then follows that both $x \in X$ and $|X|=2$. Thus, assume that $X=\{x, y\}$.

For case (i): either $x \|_{Q^{x, m}} y$, then $y=m$, and thus $Q$ has $m$ for greatest element, this contradicts that $Q$ is indecomposable. Or $y<_{Q^{x, m}} x$, then $D_{Q^{x, m}}(y)=$ $V\left(Q^{x, m}\right)-\{x, m, y\}$, and thus, since $D_{Q^{x, m}}(y)=D_{Q}(y)$ and $y \neq m$, it follows that $D_{Q}(y)=V(Q)-\{m, y\}$. But now, the assumption about the cardinality of $D_{Q}(m)$ implies either that $m$ is the greatest element of $Q$, or that $D_{Q}(m)=D_{Q}(y)$ : each of these cases contradicts that $Q$ is indecomposable. 
For case (ii): either $x \|_{Q^{\prime}} y$, in which case we have that $y \in \operatorname{Min}\left(Q^{\prime}\right)$ and that $U_{Q}^{i m}(y)=U_{Q^{\prime}}^{i m}(y)=U_{Q^{\prime}}^{i m}(x)=\{m\}$. But, since $y \in \operatorname{Min}\left(Q^{\prime}\right)-\{x\}$ and $\operatorname{Min}(Q)=$ $\operatorname{Min}\left(Q^{\prime}\right)-\{x\}$, we obtain a contradiction with the assumption about $m$. Or, $x<_{Q^{\prime}} y$ and then $y=m$. Thus, since $D_{Q^{\prime}}(x)=\emptyset$, it follows that $D_{Q}(m)=D_{Q^{\prime}}(m)-$ $\{x\}=D_{Q^{\prime}}(x)-\{m\}=\emptyset$. Consequently, $m$ is an isolated element in $Q$, this contradicts that $Q$ is indecomposable.

For case (iii): Assume that $U_{Q}(p)=\{m\}$. Either $x \|_{Q^{\prime}} y$, then we have that $y \in$ $\operatorname{Max}\left(Q^{\prime}\right)$ and that $D_{Q}(y)=D_{Q^{\prime}}(y)=D_{Q^{\prime}}(x)=\{p\}$, consequently $y=m$. It follows that $D_{Q}(m)-\{p\}=\emptyset$. Since $p \in \operatorname{Min}(Q)$ we have that $D_{Q}(m)-\{p\}=D_{Q}(p)-$ $\{m\}$. Since, by definition, $U_{Q}(p)-\{m\}=\emptyset$ and since $m \in \operatorname{Max}(Q)$ it follows that $U_{Q}(m)-\{p\}=U_{Q}(p)-\{m\}$ : this implies that $\{m, p\}$ is a non-trivial interval of $Q$ and this is impossible. Or $y<{ }_{Q^{\prime}} x$, then $y=p$, and then, it follows that $U_{Q}(p)=U_{Q^{\prime}}(p)-\{x\}=U_{Q^{\prime}}(x)-\{p\}=U_{Q^{\prime}}(x)=\emptyset$. Thus, $p$ is an isolated element in $Q$, this contradicts that $Q$ is indecomposable.

Lemma 5.2 Let $P$ be an indecomposable order, let $Q$ be any order, and let $m \in$ $\operatorname{Max}(Q)$. For any $x \notin V(Q)$, if $P$ does not embed into $Q$ but embeds into $Q^{x, m}$, then $|\operatorname{Max}(P)|=2$ and $P$ has a max dominating element.

Proof. Assume that $P$ embeds into $Q^{x, m}$ by $\psi$. Then, since $P$ does not embed into $Q$, we have that $\psi^{-1}(x)$ exists. Consequently, from Fact 2.1 , it follows that for all $y \in V(P)-\left\{\psi^{-1}(x)\right\}$ we have $y<_{P} \psi^{-1}(x)$ whenever $y \neq \psi^{-1}(m)$. But, since $P$ is indecomposable, it follows that $\psi^{-1}(m)$ exists, this gives the lemma.

Theorem 5.3 If $\mathcal{C}$ is the class of indecomposable orders, then any order $P$ in $\mathcal{C}$, on at least 6 elements, is $\mathcal{C}$-extensible.

Proof. Let $P$ be an indecomposable order on at least 6 elements, let $Q$ be any indecomposable order in which $P$ does not embed, and let $x \notin V(Q)$. By contradiction assume that $P$ embeds into every indecomposable extension of $Q$. Let $m_{a} \in \operatorname{Max}(Q)$ and let $m_{i} \in \operatorname{Min}(Q)$ having respectively, a predecessors set and a successors set of maximal cardinality. By Lemma 5.1 (i), applied to $Q$ and to $Q^{d}$, we obtain that $Q^{x, m_{a}}$ and $Q_{x, m_{i}}$ are indecomposable extensions of $Q$. Consequently, we can use Lemma 5.2 with $P, Q$ and $Q^{x, m_{a}}$ : this implies that $\operatorname{Max}(P)=\left\{\operatorname{map}_{1}\right.$, map $\left._{2}\right\}$ and that, without loss of generality, $D_{P}\left(\operatorname{map}_{1}\right)=V(P)-\operatorname{Max}(P)$. Again, we can use Lemma 5.2 with $P^{d}, Q^{d}$ and $Q_{x, m_{i}}$ : this implies that $\operatorname{Min}(P)=\left\{\right.$ mip $_{1}$, mip $\left._{2}\right\}$ and that, without loss of generality, $U_{P}\left(\operatorname{mip}_{1}\right)=V(P)-\operatorname{Min}(P)$.

Now, assume that there exists $q \in \operatorname{Min}(Q)$ such that $U_{Q}(q)=\{m q\}$. Let $Q^{\prime}=\left(V(Q) \cup\{x\},<_{Q} \cup\{(q, x)\}\right)$, then, by Lemma 5.1 (iii), $Q^{\prime}$ is an indecomposable extension of $Q$. Thus, assume that $P$ embeds into $Q^{\prime}$ by $\phi$. Then, since $P$ does not embed into $Q, \phi^{-1}(x)$ exists, and, by Property 2.1 (iii), it follows that $\phi^{-1}(x) \in \operatorname{Max}(P)$. Then, since $P$ is connected, Fact 2.1 implies that $\phi^{-1}(q)$ exists. But now, again from Fact 2.1 we have that $\left|U_{P}\left(\phi^{-1}(q)\right)\right| \leq\left|U_{Q^{\prime}}(q)\right|=2$, from Property 2.1 (iii) we obtain that $\phi^{-1}(q) \in \operatorname{Min}(P)$, and from Property 2.1 (ii) it follows 
that $D_{P}^{i m}\left(\phi^{-1}(x)\right)=\left\{\phi^{-1}(q)\right\}$. Consequently, we have $D_{P}\left(\phi^{-1}(x)\right)=\left\{\phi^{-1}(q)\right\}$ and then $\phi^{-1}(q)=$ mip $_{1}$, this implies that $|V(P)| \leq 4$ : a contradiction.

If we assume that there exists $q \in \operatorname{Max}(Q)$, such that $D_{Q}(q)=\{m q\}$, we obtain a similar contradiction with $Q^{\prime}=\left(V(Q) \cup\{x\},<_{Q} \cup\{(x, q)\}\right)$.

Consequently, for any $\operatorname{miq} \in \operatorname{Min}(Q)$ and for any $\operatorname{maq} \in \operatorname{Max}(Q)$, by Lemma 5.1(ii), it follows that the orders $Q_{m i q}=\left(V(Q) \cup\{x\},<_{Q} \cup\{(\right.$ miq,$\left.x)\}\right)$ and $Q_{\text {maq }}=\left(V(Q) \cup\{x\},<_{Q} \cup\{(x, m a q)\}\right)$ are indecomposable extensions of $Q$. Thus, on the one hand, $P$ embeds into $Q_{\text {miq }}$ by $\psi$ and we have both $\psi^{-1}(x) \in \operatorname{Max}(P)$ and $\left|D_{P}\left(\psi^{-1}(x)\right)\right|=1$. On the other hand, $P$ embeds into $Q_{m a q}$ by $\phi$, and we have both $\phi^{-1}(x) \in \operatorname{Min}(P)$ and $\left|U_{P}\left(\phi^{-1}(x)\right)\right|=1$. It follows that the unique non max (respectively, non min) dominating element of $P$ has for single predecessor (respectively, successor) the unique min (respectively, max) dominating element of $P$. Therefore $V(P) \backslash(\operatorname{Max}(P) \cup \operatorname{Min}(P))$ is a non-trivial interval of $P$ : a contradiction.

To complete the study of faithful extensions on the class $\mathcal{C}$ of indecomposable orders, notice that, since any indecomposable order has a suborder isomorphic to the only -up to isomorphism- indecomposable order on 4 elements (for example $(\{1,3,2,4\},\{(1,3),(2,4),(2,3)\}))$, it is then sufficient to consider indecomposable orders on 5 elements.

Firstly, following the first part of the proof of Theorem 5.3, we obtain that orders with more than two maximal or two minimal elements are $\mathcal{C}$-extensible.

Secondly, since it is always possible to extend any indecomposable order by adding either a minimal or a maximal pendant (a minimal pendant is any minimal element with a unique immediate successor), orders with neither minimal nor maximal pendant are $\mathcal{C}$-extensible.

It is then a matter of routine to check that the only -up to isomorphism- indecomposable order on 5 elements having a pendant element, two maximal elements and two minimal elements is isomorphic to

$$
P=(\{1,3,2,4, b\},\{(1,3),(2,4),(2,3),(2, b),(b, 3)\})
$$

(see Figure 1). If we think that $P$ is $\mathcal{C}$-extensible we do not have a proof.

\section{Conclusion}

If for the class $\mathcal{H}_{k}$ we are unable to solve the general case, nevertheless we can strengthen a little the result of Fraïssé and Hazim-Sharif [6]. Indeed we show that every order (of height $k$ ) having at least one level of its rank decomposition without a maximal element is $\mathcal{H}_{k}$-extensible. Therefore, it only remains unsolved the case of orders in $\mathcal{H}_{k}$, with $1<k$, having a maximal element in each level of its rank decomposition and such that its unique connected component on at least two elements has both a max dominating and a min dominating element.

Proposition 6.1 Any order $P$ in $\mathcal{H}_{k}$, such that $\mid\left\{i \in[k+1]: \mathcal{R}_{P}(i-1) \cap \operatorname{Max}(P) \neq\right.$ $\emptyset\} \mid \neq k+1$, is $\mathcal{H}_{k}$-extensible. 
Proof. We proceed by contradiction. We assume that there exists $Q$ in $\mathcal{H}_{k}$, such that $P$ does not embed into $Q$ but embeds into every sharp extension of $Q$ belonging to $\mathcal{H}_{k}$. Let $x \notin V(Q)$, and, for $i \in\{0, \ldots, k\}$, let $Q_{i}=\left(V(Q) \cup\{x\},<_{Q} \cup\{(y, x)\right.$ : $\left.\left.y \in \bigcup_{j=0}^{i-1} \mathcal{R}_{Q}(i)\right\}\right)$. Notice that $Q_{i} \in \mathcal{H}_{k}$, and thus assume that $P$ embeds into $Q_{i}$ by $\phi_{i}$. Since $P$ does not embed into $Q$, for every $i \in\{0, \ldots, k\}$ we have that $\phi_{i}^{-1}(x)$ exists, and, by Property 2.1 (iii), it follows that $\phi_{i}^{-1}(x) \in \operatorname{Max}(P)$. Since, by Fact 2.1, for every $p \in V(P)$ we have that $\operatorname{rank}_{P}(p) \leq \operatorname{rank}_{Q}(\phi(p))$. Then to prove that, for every $i \in\{0, \ldots, k\} \operatorname{rank}_{P}\left(\phi_{i}^{-1}(x)\right)=i$, it is sufficient to show that $i \leq \operatorname{rank}_{P}\left(\phi_{i}^{-1}(x)\right)$. Let $c=\left(x_{0}, \ldots, x_{k}\right)$ be a maximal sized chain of $P$. Then, since $h\left(Q_{i}\right)=k,\left(\phi_{i}\left(x_{0}\right), \ldots, \phi_{i}\left(x_{k}\right)\right)$ is also a maximal sized chain of $Q_{i}$ and thus $\operatorname{rank}_{Q}\left(\phi\left(x_{j}\right)\right)=j$ for $j \in\{0, \ldots, k\}$. Consequently, $\left(x_{0}, \ldots, x_{i-1}, \phi_{i}^{-1}(x)\right)$ is a chain of $P$ and thus $i \leq_{\mathbb{N}} \operatorname{rank}_{P}\left(\phi_{i}^{-1}(x)\right)$.

As already mentioned in Section 4, we believe that the challenging class of modular lattices is worth to be studied. Likewise, the class of tree-like orders (or poset tree orders see Trotter [14] on page 113 and page 117) seems interesting to study. Indeed, the tree-like order's sharp extensions, being tree-like, are obtained by adding a new element having a unique lower (respectively, upper) cover, say $y$, and then having for upper (respectively, lower) covers any subset of the original set of upper (respectively, lower) covers of $y$. Now, on the one hand, the fact that these sharp extensions are highly targeted, and are simple to characterize, could lead to conclude that the extensibility can be easily obtained. But, on the other hand, the fact that these sharp extensions are obtained by modifications having a strongly local character, makes the problem harder to solve. In fact, we think that, as soon as a class of connected orders is stable under pendant extensions, this class is then extensible. When, a pendant extension of an order is any extension obtained by adding a new maximal (respectively, minimal) element having a unique lower (respectively, upper) cover.

Notice that such a result would also imply that the class of connected planar orders (see Trotter [14] on page 66) is extensible.

\section{Acknowledgements}

The authors thank a referee for his/her helpful suggestions which substantially helped to improve the quality of the paper.

\section{References}

[1] G. Birkhoff, Lattice theory, American Mathematical Society Colloquium Publications Volume XXV, 1967.

[2] B. A. Davey and H. A. Priestley, Introduction to Lattices and Order, Cambridge University Press, 1990.

[3] R. Fraïssé, Sur la comparaison des types de relation, C.R. Acad. Sci. Paris, t. 226 (1948), 987-988. 
[4] R. Fraïssé, Abritement entre relations et spécialement entre chaînes, Mathematica, Vol. V (INDAM, Rome, 1969/70) (1971), 203-251.

[5] R. Fraïssé, Theory of Relations, revised edition, Studies in Logic and the Foundations of Mathematics, Vol. 145, 2000.

[6] R. Fraïssé and W. Hazim-Sharif, L'extension respectueuse à hauteur constante entre posets finis, C.R. Acad. Sci. Paris, t. 316, Série 1 (1993), 637-642.

[7] T. Gallai, Transitiv orientierbare Graphen, Acta Math. Acad. Sci. Hungar. 18 (1967), 25-66.

[8] G.-B. Guenver, J. Leblet and J.-X. Rampon, Chain Dominated Orders, Order 23 (2006), 109-127.

[9] M. Habib, Substitution des structures combinatoires, théorie et algorithmes, Thèse d'État, Université Pierre et Marie Curie, 1981.

[10] P. Ille, La décomposition intervallaire des structures binaires, Gazette des Mathématiciens 104 (2005), 39-58.

[11] P. Jullien, Sur un théorème d'extension dans la théorie des mots, C.R. Acad. Sci. Paris, t. 262, Série A (1968), 851-854.

[12] D. Kelly, Comparability graphs, in Graphs and orders (1985), 3-40.

[13] B. S. W. Schröder, Ordered sets, An introduction, Birkhäuser Boston, 2003.

[14] W. T. Trotter, Combinatorics and partially ordered sets: dimension theory, The John Hopkins University Press, 1992.

(Received 18 June 2015; revised 24 July 2017) 